\title{
Toward group theory operators for tmLQCD hadrons
}

\section{Derek Harnett}

Department of Physics, University College of the Fraser Valley, Abbotsford, BC, Canada, V2S $7 M 8$

E-mail: derek.harnett@ucfv.ca

\section{Randy Lewis and Robert G. Petry*}

Department of Physics, University of Regina, Regina, SK, Canada, S4S OA2

E-mail: randy.lewis@uregina.ca, rob.petry@uregina.ca

Extraction of the mass spectrum from twisted mass lattice QCD is facilitated by respecting the octahedral group of rotations and accommodating the broken parity and flavor symmetries of the theory. In this work, group theory meson operators adapted to these constraints are constructed for the special case of quark and antiquark fields at a common lattice site, connected by extended gauge field paths.

XXIVth International Symposium on Lattice Field Theory

July 23-28, 2006

Tucson, Arizona, USA

\footnotetext{
*Speaker.
} 


\section{Introduction}

\subsection{Motivation}

Twisted mass lattice QCD (tmLQCD) offers an efficient mechanism for eliminating unphysical zero modes thereby admitting calculations at lighter quark masses[1]. At maximal twist one has $O(a)$ improvement[2]. Lighter quark mass calculations require improved statistics for hadron mass resolution. This fact and the need to disentangle physical states with the same quantum numbers may in part be accomplished through the creation of more operators that represent the channel in question. The creation of more elaborate operators also allows for the study of hybrid and exotic mesons.

The violation of parity by the twisted mass action causes many operators to be twist angle dependent resulting in correlator contamination by opposite parity states when not at maximal twist. This suggests seeking a class of twist independent operators from which the physical parity of an operator is readily deduced.

Operators with displaced quarks have been constructed using group theoretical techniques but their usage necessarily requires the calculation of quark propagators from multiple sources. (See, for example, Refs. [3, 4, 5].) Consideration of the operators available through gluonic extension alone therefore is calculationally expedient.

\subsection{Lattice Symmetry Group}

While parity $(P)$ and charge conjugation $(C)$ may be conserved by lattice actions, the continuous rotational symmetry of nature is broken and one requires operators adapted to the symmetry group of the lattice. For mesons this is the octahedral group $O$ with 24 elements. The direct product of the parity, charge conjugation, and octahedral groups is denoted $O^{P C}$.

For an operator adapted to the representation $\Lambda^{P C}$, where $\Lambda \in\left\{A_{1}, A_{2}, E, T_{1}, T_{2}\right\}$ is an irreducible representation (irrep) of $O$ and $P, C \in\{+,-\}$, one identifies the possible physical states $J^{P C}$ to which it corresponds using Table 1 which shows the number of copies $n_{\Lambda}^{J}$ of irrep $\Lambda$ to which the continuum $S U(2)$ irrep $J$ subduces[6]. An operator transforming as the irrep $E^{+-}$could

\begin{tabular}{|l|ccccc||c|ccccc|}
\hline$J$ & $A_{1}$ & $A_{2}$ & $E$ & $T_{1}$ & $T_{2}$ & $J$ & $A_{1}$ & $A_{2}$ & $E$ & $T_{1}$ & $T_{2}$ \\
\hline 0 & 1 & 0 & 0 & 0 & 0 & 3 & 0 & 1 & 0 & 1 & 1 \\
1 & 0 & 0 & 0 & 1 & 0 & 4 & 1 & 0 & 1 & 1 & 1 \\
2 & 0 & 0 & 1 & 0 & 1 & 5 & 0 & 0 & 1 & 2 & 1 \\
\hline
\end{tabular}

Table 1: Subduction of continuum irreps to those of the lattice symmetry group.

have spin content $\left(J^{P C}=2^{+-}, 4^{+-}, 5^{+-}, \ldots\right)$. In theory one needs many operators of each $\Lambda^{P C}$ to resolve the many physical spins $J^{P C}$ in the tower of states to which $\Lambda^{P C}$ corresponds.

\subsection{Operator Building Blocks}

One may construct zero-momentum operators transforming as irreps of $O^{P C}$ from the space of operators spanned by

$$
M_{j, k, a, b}(t)=\sum_{\mathbf{x}} \bar{\psi}_{a}(x) U_{j}(x) U_{k}(x+\hat{j}) U_{-j}(x+\hat{j}+\hat{k}) U_{-k}(x+\hat{k}) \psi_{b}(x),
$$


where $j, k= \pm 1, \pm 2, \pm 3 ; j \neq k$ and $a$ and $b$ are spinor indices for a total of $24 \times 16=384$ operators. See Figure 1 for a diagrammatic representation. Superpositions of such operators for meson

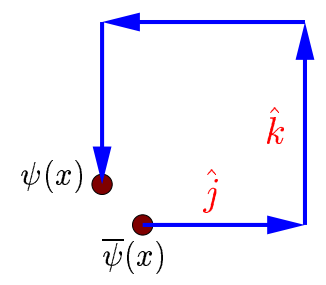

Figure 1: Diagrammatic representation of the building block operator.

spectra analysis have been suggested and used previously in special cases[7, \%, 3, 9]. Evaluation of correlators constructed from operators of this form requires only calculation of propagators from a single source. Construction of operators transforming as irrep $\Lambda^{P C}$ is facilitated by observing that one can effectively consider the transformation of the spinor and link paths independently and then combine them via octahedral Clebsch-Gordan coefficients, as shown in Sections \&, and 4

\section{Spinor Contribution to Group Structure}

The contribution to the group structure due to spinor indices is determined by the 16 bilinears $\bar{\psi} F \psi$, where $F$ represents one of sixteen $4 \times 4$ matrices, $\left\{I, \gamma_{5}, \gamma_{4}, \gamma_{4} \gamma_{5}, \gamma_{i}, \gamma_{i} \gamma_{5}, \sigma_{4 i}, \varepsilon_{i j k} \sigma_{j k}\right\}$. The first four bilinears are scalars while the last four three-index objects are vectors under the rotation group in the Euclidean continuum.

Parity $(P)$ and charge conjugation $(C)$ of the bilinears are identified via

$$
\begin{array}{cc}
\mathscr{C} \mathscr{C}^{\dagger}=\left(\bar{\psi} C^{\dagger}\right)^{T} & \mathscr{P} \psi \mathscr{P}^{\dagger}=\gamma_{4} \psi \\
\mathscr{C} \bar{\psi} \mathscr{C}^{\dagger}=-(C \psi)^{T} & \mathscr{P} \bar{\psi} \mathscr{P}^{\dagger}=\bar{\psi} \gamma_{4}
\end{array},
$$

where $C$ is the matrix implementing charge conjugation.

To classify the bilinears into irreps of $O$ one uses its character table found in Table 2] Since

\begin{tabular}{|c|rrrrr|}
\hline$\Lambda \backslash \xi$ & $E$ & $3 C_{4}^{2}$ & $8 C_{3}$ & $6 C_{4}$ & $6 C_{2}$ \\
\hline$A_{1}$ & 1 & 1 & 1 & 1 & 1 \\
$A_{2}$ & 1 & 1 & 1 & -1 & -1 \\
$E$ & 2 & 2 & -1 & 0 & 0 \\
$T_{1}$ & 3 & -1 & 0 & 1 & -1 \\
$T_{2}$ & 3 & -1 & 0 & -1 & 1 \\
\hline
\end{tabular}

Table 2: Character table giving $\chi(\xi)$ for octahedral group $O$.

the bilinears are scalars and vectors in the continuum it follows that their respective spans are also invariant under $O$. By inspection it is straightforward to show that the character table for the scalar and vector bilinears is identical with that of $A_{1}$ and $T_{1}$ respectively. The multiplicity $n_{\Lambda}^{J}$ of irrep $\Lambda$ in the representation $J$ is given by

$$
n_{\Lambda}^{J}=\frac{1}{g_{o}} \sum_{\xi} p_{\xi} \chi^{(\Lambda)}(\xi)^{*} \chi^{(J)}(\xi),
$$


where $p_{\xi}$ is the number of elements in class $\xi$ and $g_{o}$ is the order of the octahedral group[6]. It follows trivially by this formula that the scalar and vector bilinears form the basis of $A_{1}$ and $T_{1}$ irreps respectively. Furthermore it may be verified that the $i^{\text {th }}$ vector bilinear component transforms as the $i^{t h}$ row for our choice of matrix representation $\Gamma^{\left(T_{1}\right)}(R)$.

Hence the reduction of the spinor structure of our operators is given in Table 3 , where now each bilinear may be classified uniquely by its irrep $\Lambda^{P C}$, row $\lambda$, and irrep multiplicity index $\alpha$.

\begin{tabular}{|c|c|c|c||c|c|c|c|}
\hline$F$ & $\Lambda^{P C}$ & $\lambda$ & $\alpha$ & $F$ & $\Lambda^{P C}$ & $\lambda$ & $\alpha$ \\
\hline$I$ & $A_{1}^{++}$ & 1 & 1 & $\gamma_{i}$ & $T_{1}^{--}$ & $i$ & 1 \\
$\gamma_{5}$ & $A_{1}^{-+}$ & 1 & 1 & $\gamma_{i} \gamma_{5}$ & $T_{1}^{++}$ & $i$ & 1 \\
$\gamma_{4}$ & $A_{1}^{+-}$ & 1 & 1 & $\sigma_{4 i}$ & $T_{1}^{--}$ & $i$ & 2 \\
$\gamma_{4} \gamma_{5}$ & $A_{1}^{-+}$ & 1 & 2 & $\varepsilon_{i j k} \sigma_{j k}$ & $T_{1}^{+-}$ & $i$ & 1 \\
\hline
\end{tabular}

Table 3: Octahedral symmetries of the spinor bilinears. Red and green entries are twist angle invariant for charged and neutral mesons respectively as shown in Section 5.1 .

\section{Link Contribution to Group Structure}

Define the gauge link part of our operators in equation (1.1) by

$$
U_{j, k}(x) \equiv U_{j}(x) U_{k}(x+\hat{j}) U_{-j}(x+\hat{j}+\hat{k}) U_{-k}(x+\hat{k}),
$$

where $j, k= \pm 1, \pm 2, \pm 3 ; j \neq k$. The parity and charge conjugation contributions due to this link structure can then be taken into account by defining the $P C$-adapted superpositions,

$$
U_{i, j}^{P C}=U_{j, k}+P U_{-j,-k}+C U_{k, j}+P C U_{-k,-j}
$$

where $k$ is defined via $\hat{k}=\hat{i} \times \hat{j}$ and we have suppressed an overall normalization factor of $1 / 2$. Diagrammatically, $U_{i, j}^{P C}$ is shown in Figure 2. For fixed $P C$ the space spanned by $U_{i, j}^{P C}$ is invariant

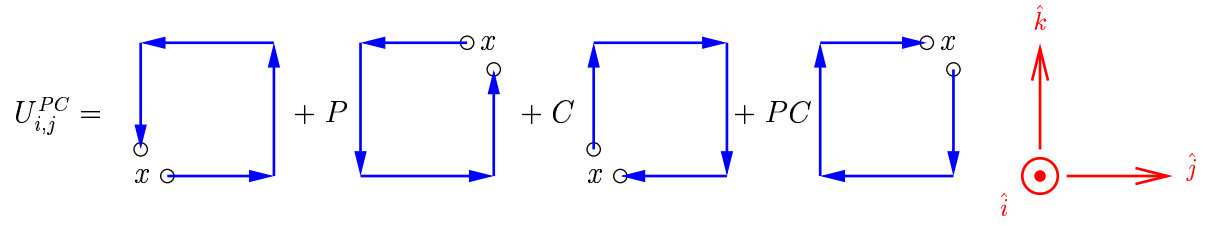

Figure 2: $P C$-symmetrized basis elements for gauge field links.

under $O$ and will generate a representation $U^{P C}$ of the group. A basis for the six-dimensional space may be found by restricting $(i, j)$ to $\{(1,2),(1,3),(2,3),(2,1),(3,1),(3,2)\}$ and this will in turn give the particular matrix representation $\Gamma_{(i, j)(m, n)}^{\left(U^{P C}\right)}(R)$. The character table for $U^{P C}$ giving $\chi^{\left(U^{P C}\right)}(\xi)$ is shown in Table 4 . Use of Formula (2.2) reduces $U^{P C}$ to irreps $\Lambda^{P C}$ of $O^{P C}$ as shown in Table 5 .

The symmetrized link fields corresponding to this reduction are 


\begin{tabular}{|lllll|}
\hline$E$ & $3 C_{4}^{2}$ & $8 C_{3}$ & $6 C_{4}$ & $6 C_{2}$ \\
\hline 6 & $2 P$ & 0 & 0 & $(P+1) C$ \\
\hline
\end{tabular}

Table 4: Character table for representation $U^{P C}$ induced by $U_{i, j}^{P C}$.

\begin{tabular}{|c|ccccc|}
\hline$P C$ & $A_{1}^{P C}$ & $A_{2}^{P C}$ & $E^{P C}$ & $T_{1}^{P C}$ & $T_{2}^{P C}$ \\
\hline++ & 1 & 0 & 1 & 0 & 1 \\
+- & 0 & 1 & 1 & 1 & 0 \\
-+ & 0 & 0 & 0 & 1 & 1 \\
-- & 0 & 0 & 0 & 1 & 1 \\
\hline
\end{tabular}

Table 5: Reduction of $U^{P C}$ to irreps $\Lambda^{P C}$ of $O^{P C}$.

$$
U_{\lambda}^{\Lambda^{P C}}(x)=\sum_{(i, j)} \frac{\sum_{R \in O} \Gamma_{(i, j)(m, n)}^{\left(U^{P C}\right)}(R) \Gamma_{\lambda \mu}^{(\Lambda)}(R)^{*}}{\left[\frac{g_{O}}{d_{\Lambda}} \sum_{R \in O} \Gamma_{(m, n)(m, n)}^{\left(U^{P C}\right)}(R) \Gamma_{\mu \mu}^{(\Lambda)}(R)^{*}\right]^{\frac{1}{2}}} U_{i, j}^{P C}(x),
$$

where $(m, n)$ and $\mu$ are chosen so the denominator does not vanish and the accessible $\Lambda^{P C}$ are chosen from Table 5. (See Ref. [10, page 74].) Here $\Gamma_{\lambda \mu}^{(\Lambda)}(R)$ is our matrix representation for the irrep $\Lambda$. The $U_{\lambda}^{\Lambda^{P C}}(x)$ transform as the row $\lambda$ of irrep $\Lambda^{P C}$ and are uniquely identified by them.

\section{Total Representation Reduction and Operator Construction}

\subsection{Reduction of Direct Product Representations}

Having classified the spinor and link components of our operators into irreps of $O^{P C}$, it remains to combine them and reduce all possible direct product representations. Parity and charge conjugation for the product representations are given by

$$
P=P_{f} P_{u} \quad C=C_{f} C_{u}
$$

where $f$ corresponds to the fermionic part and $u$ corresponds to the gauge part. Noting that the character of $\Lambda_{1} \otimes \Lambda_{2}$ satisfies $\chi^{\left(\Lambda_{1} \otimes \Lambda_{2}\right)}(\xi)=\chi^{\left(\Lambda_{1}\right)}(\xi) \chi^{\left(\Lambda_{2}\right)}(\xi)$ and using Formula (2.2), one may reduce each direct product into irreps of $O$. As such the 384-dimensional representation generated by the space spanned by $M_{j, k, a, b}(t)$ may be reduced into irreps of $O^{P C}$ as shown in Table 6 . Note that all possible lattice irreps are accessible, not just the $A_{1}^{P C}$ and $T_{1}^{P C}$ of simpler local operators.

\subsection{Operator Construction Using Clebsch-Gordan Coefficients}

The construction of the lattice-symmetrized operators themselves is accomplished by combining the spinor and link operator components $F_{\lambda}^{\Lambda^{P C}, \alpha}$ and $U_{\lambda}^{\Lambda^{P C}}$ using the same formulae for parity and charge conjugation given in Equation (4.1) and by combining the irreps of $O$ using ClebschGordan (C-G) coefficients for $\Lambda_{1} \otimes \Lambda_{2}$. The simplicity of the possible direct product representations 


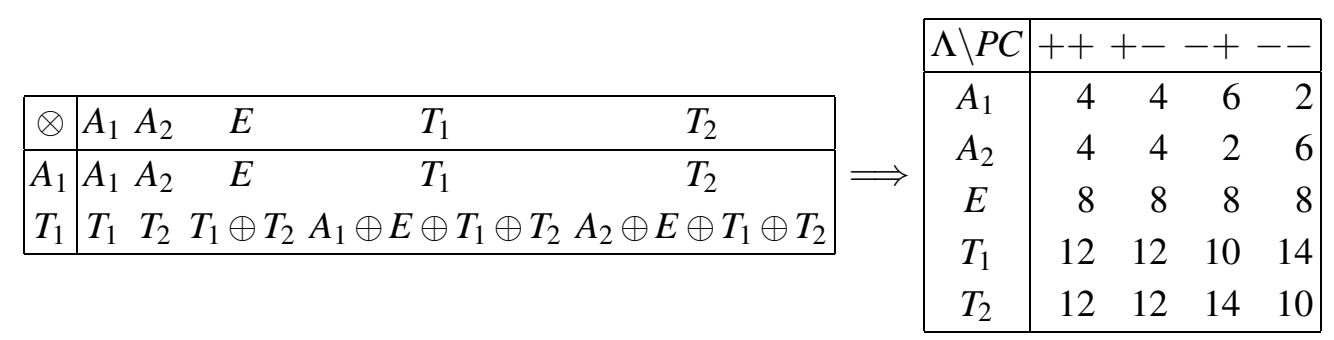

Table 6: Reduction of representation generated by span of $M_{j, k, a, b}(t)$ into $O^{P C}$ irreps.

allows one to use

$$
\left(\begin{array}{cc|c}
\Lambda_{1} & \Lambda_{2} & \Lambda \\
\lambda_{1} & \lambda_{2} & \lambda
\end{array}\right)=\frac{\sum_{R \in O} \Gamma_{\lambda_{1} \mu_{1}}^{\left(\Lambda_{1}\right)}(R) \Gamma_{\lambda_{2} \mu_{2}}^{\left(\Lambda_{2}\right)}(R) \Gamma_{\lambda \mu}^{(\Lambda)}(R)^{*}}{\left[\frac{g O}{d_{\Lambda}} \sum_{R \in O} \Gamma_{\mu_{1} \mu_{1}}^{\left(\Lambda_{1}\right)}(R) \Gamma_{\mu_{2} \mu_{2}}^{\left(\Lambda_{2}\right)}(R) \Gamma_{\mu \mu}^{(\Lambda)}(R)^{*}\right]^{\frac{1}{2}}}
$$

to determine the C-G coefficients. (See Ref. [10, page 74].) Here $\mu, \mu_{1}$, and $\mu_{2}$ are chosen so the denominator does not vanish. The lattice-symmetrized operators, $M_{\lambda}^{\Lambda^{P C}}(t)$, are then given by

$$
M_{\lambda}^{\Lambda^{P C}, \Lambda_{f}^{P_{f} C_{f}}, \alpha_{f}, \Lambda_{u}^{P_{u} C_{u}}}(t)=\sum_{\mathbf{x}} \sum_{\lambda_{f}, \lambda_{u}}\left(\begin{array}{cc|c}
\Lambda_{f} & \Lambda_{u} & \Lambda \\
\lambda_{f} & \lambda_{u} & \lambda
\end{array}\right) \bar{\psi}(x) F_{\lambda_{f}}^{\Lambda_{f}^{P_{f} C_{f}}, \alpha_{f}} U_{\lambda_{u}}^{\Lambda_{u}^{P_{u} C_{u}}}(x) \psi(x),
$$

where the allowed irreps for the spinor bilinear and link components are determined by Tables 3 and 5 . These fix $P$ and $C$ for the operator while the irreps $\Lambda$ of $O$ are those allowed by the C-G series in Table 6 . Dirac indices on $F$ and color indices on $U$ and both indices on the spinors have been suppressed.

\section{Twisted Mass Invariant Operators}

\subsection{Operators with Definite Parity}

Consider tmLQCD for a quark doublet. A change of basis (by twist angle $\omega$ ) gives

$$
\left(\begin{array}{l}
u^{\prime} \\
d^{\prime}
\end{array}\right)=\exp \left(i \omega \gamma_{5} \tau_{3}\right)\left(\begin{array}{l}
u \\
d
\end{array}\right)
$$

which leads to mixing among some, but not all, quark bilinears. For example,

$$
\bar{u}^{\prime} \gamma_{i} d^{\prime}=\bar{u} \gamma_{i} d \cos \omega+\bar{u} \gamma_{i} \gamma_{5} d \sin \omega
$$

couples to both vector and axial mesons unless $\omega$ is carefully tuned, but

$$
\bar{u}^{\prime} \sigma_{4 i} d^{\prime}=\bar{u} \sigma_{4 i} d
$$

couples to the vector and never to the axial. Explicit calculation shows that tmLQCD splits the results of Section 2 into two sets according to flavor. Structures in red in Table 3 are only independent of twist angle for charged mesons, while green structures are only independent of twist angle for neutral mesons. Despite these restrictions, one finds that all $\Lambda^{P C}$ combinations can still be obtained by factoring in some nontrivial link structure. Combining the twist-invariant spinor structures from Table 3 with the link structure in Table 5 results in operators of the irreps outlined in Table 7being accessible. 


charged mesons*
\begin{tabular}{|c|cccc|}
\hline$\Lambda \backslash P C$ & ++ & +- & -+ & - \\
\hline$A_{1}$ & 3 & 1 & 3 & 1 \\
$A_{2}$ & 1 & 3 & 1 & 3 \\
$E$ & 4 & 4 & 4 & 4 \\
$T_{1}$ & 5 & 7 & 5 & 7 \\
$T_{2}$ & 7 & 5 & 7 & 5 \\
\hline
\end{tabular}

neutral mesons
\begin{tabular}{|c|cccc|}
\hline$\Lambda \backslash P C$ & ++ & +- & -+ & - \\
\hline$A_{1}$ & 1 & 3 & 3 & 1 \\
$A_{2}$ & 3 & 1 & 1 & 3 \\
$E$ & 4 & 4 & 4 & 4 \\
$T_{1}$ & 7 & 5 & 5 & 7 \\
$T_{2}$ & 5 & 7 & 7 & 5 \\
\hline
\end{tabular}

Table 7: Twist invariant irreps for charged and neutral mesons respectively. *It is to be noted here that charged mesons are really only eigenstates of g-parity with eigenvalue $G=C(-1)^{I}$, but we use $C$ for simple correspondence with the previous arguments.

\subsection{Preserving Parity in the Correlators}

Building states of definite $\Lambda^{P C}$ might seem insufficient for tmLQCD, since the theory does not preserve $P$. However, tmLQCD does conserve a "twisted parity", $\tilde{P}$, defined by the product of $P$ and $(\omega \rightarrow-\omega)$. Therefore, states of definite twisted parity are automatically preserved in any correlation function. Since our quark bilinears are independent of $\omega, P$ and $\tilde{P}$ are equivalent for these bilinears.

\section{Acknowledgements}

This work was supported in part by the Natural Sciences and Engineering Research Council of Canada, the Canada Foundation for Innovation, the Canada Research Chairs Program and the Government of Saskatchewan.

\section{References}

[1] R. Frezzotti, P. A. Grassi, S. Sint, and P. Weisz. JHEP 08, 058 (2001) [arXiv:hep-lat/0101001].

[2] R. Frezzotti and G. C. Rossi. JHEP 08, 007 (2004) [arXiv:hep-lat/0306014].

[3] P. Lacock, C. Michael, P. Boyle, and P. Rowland. Phys. Rev. D54, 6997 (1996) [arXiv:hep-lat/9605025].

[4] S. Basak et al. Phys. Rev. D72, 094506 (2005) [arXiv:hep-lat/0506029].

[5] S. Basak et al. Phys. Rev. D72, 074501 (2005) [arXiv:hep-lat/0508018].

[6] R. C. Johnson. Phys. Lett. B114, 147 (1982).

[7] J. E. Mandula. Phys. Lett. B135, 155 (1984).

[8] I. H. Jorysz and C. Michael. Nucl. Phys. B302, 448 (1988).

[9] C. W. Bernard et al. Phys. Rev. D56, 7039 (1997) [arXiv:hep-lat/9707008].

[10] J. F. Cornwell. Group theory in physics: An introduction. San Diego, USA: Academic (1997) 349 p. 